II presente documento viene fornito attraverso il servizio NILDE dalla Biblioteca fornitrice, nel rispetto della vigente normativa sul Diritto d'Autore (Legge n.633 del 22/4/1941 e successive modifiche e integrazioni) e delle clausole contrattuali in essere con il titolare dei diritti di proprietà intellettuale.

La Biblioteca fornitrice garantisce di aver effettuato copia del presente documento assolvendo direttamente ogni e qualsiasi onere correlato alla realizzazione di detta copia.

La Biblioteca richiedente garantisce che il documento richiesto è destinato ad un suo utente, che ne farà uso esclusivamente personale per scopi di studio o di ricerca, ed è tenuta ad informare adeguatamente i propri utenti circa i limiti di utilizzazione dei documenti forniti mediante il servizio NILDE.

La Biblioteca richiedente è tenuta al rispetto della vigente normativa sul Diritto d'Autore e in particolare, ma non solo, a consegnare al richiedente un'unica copia cartacea del presente documento, distruggendo ogni eventuale copia digitale ricevuta.

Biblioteca richiedente: Biblioteca Dipartimento Scienze di base e applicate per l'ingegneria - Sez.Fisica

Data richiesta:

05/11/2020 16:07:23

Biblioteca fornitrice: INAF - Osservatorio Astronomico di Cagliari

Data evasione: $\quad$ 05/11/2020 16:20:10

Titolo rivista/libro: $\quad$ Proceedings of SPIE

Titolo articolo/sezione: Operational experience on the generation and control of high brightness electron bunch trains at SPARC-LAB

Autore/i: $\quad$ Mostacci,Alesini, Anania

ISSN: $\quad$ 1996-756X

DOI:

Anno:

Volume:

9512

Fascicolo:

Editore:

Pag. iniziale:

Pag. finale: 


\section{Operational experience on the generation and control of high brightness electron bunch trains at SPARC-LAB}

Mostacci, A., Alesini, D., Anania, M. P., Bacci, A., Bellaveglia, M., et al.

A. Mostacci, D. Alesini, M. P. Anania, A. Bacci, M. Bellaveglia, A. Biagioni, F. Cardelli, Michele Castellano, Enrica Chiadroni, Alessandro Cianchi, M. Croia, Domenico Di Giovenale, Giampiero Di Pirro, Massimo Ferrario, Francesco Filippi, Alessandro Gallo, Giancarlo Gatti, Anna Giribono, L. Innocenti, A. Marocchino, M. Petrarca, L. Piersanti, S. Pioli, Riccardo Pompili, Stefano Romeo, Andrea Renato Rossi, V. Shpakov, J. Scifo, Cristina Vaccarezza, Fabio Villa, L. Weiwei, "Operational experience on the generation and control of high brightness electron bunch trains at SPARC-LAB," Proc. SPIE 9512, Advances in X-ray Free-Electron Lasers Instrumentation III, 95121Q (12 May 2015); doi: 10.1117/12.2182566 


\title{
Operational experience on the generation and control of high brightness electron bunch trains at SPARC-LAB
}

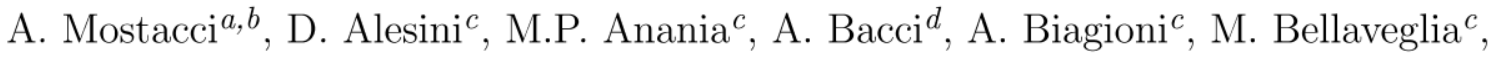

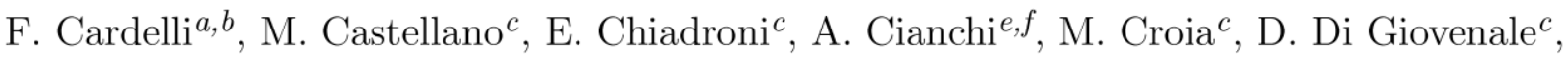

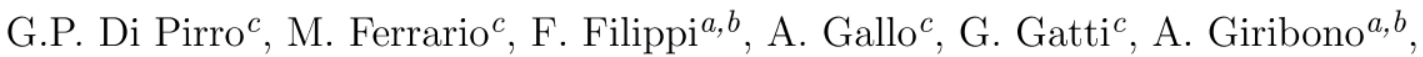 \\ L. Innocenti ${ }^{e}$, A. Marocchino ${ }^{a, b}$, M. Petrarca ${ }^{a, b}$, L. Piersanti $^{b}$, S. Pioli $^{c}$, R. Pompili $^{c}$,

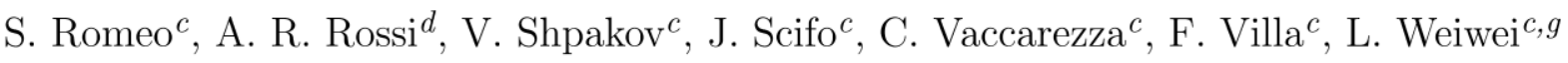 \\ ${ }^{a}$ University of Rome La Sapienza \& INFN, via Antonio Scarpa 24, 00161 Roma, Italy; \\ ${ }^{b}$ INFN-Sezione di Roma, P.le Aldo Moro 1, 00161 Roma, Italy; \\ ${ }^{c}$ INFN-LNF, Via E. Fermi, 40 - 00044 Frascati, Rome, Italy; \\ IINFN-Sezione di Milano, via Celoria 16, 20133 Milano, Italy; \\ ${ }^{e}$ University of Rome Tor Vergata, Via della Ricerca Scientifica, 1-00133 Rome, Italy; \\ fINFN-Sezione di Tor Vergata, Via della Ricerca Scientifica, 1-00133 Rome, Italy; \\ ${ }^{g}$ National Synchrotron Radiation Laboratory, University of Science and Technology of \\ China, Hefei, Anhui, 230029, China.
}

\begin{abstract}
Sub-picosecond, high-brightness electron bunch trains are routinely produced at SPARC-LAB via the velocity bunching technique. Such bunch trains can be used to drive multi-color Free Electron Lasers (FELs) and plasma wake field accelerators. In this paper we present recent results at SPARC-LAB on the generation of such beams, highlighting the key points of our scheme. We will discuss also the on-going machine upgrades to allow driving FELs with plasma accelerated beams or with short electron pulses at an increased energy.
\end{abstract}

Keywords: High brightness electrons, Free Electron Laser, Plasma accelerators, Velocity bunching

\section{INTRODUCTION}

Ps-spaced bunch trains are currently generated and manipulated in the SPARC-LAB; the production scheme of the bunch train does not rely on beam losses (and therefore it can accommodate naturally higher charge in the bunches) and the final bunch train parameters can be tuned only varying the accelerator working conditions. Both properties are interesting for several applications, such as Plasma Wake Field Acceleration (PWFA) or resonant enhancement of the $\mathrm{THz}$ radiation production ${ }^{1}$ or ultra-fast pump and probe experiments with FEL photons. ${ }^{2}$ Moreover the our scheme offers the possibility of tailoring the bunch train shape, as needed for example in PWFA.

In this paper, after an introduction reviewing the key features of our scheme (sec. 2), we will report on recent results in the manipulation of such beams, focusing on the two relevant SPARC-LAB research lines, namely multicolor FEL (sec. 3) and plasma acceleration (sec. 4).

Andrea.Mostacci@uniroma1.it

Advances in X-ray Free-Electron Lasers Instrumentation III, edited by Sandra G. Biedron Proc. of SPIE Vol. 9512, 95121Q - @ 2015 SPIE · CCC code: 0277-786X/15/\$18

doi: $10.1117 / 12.2182566$

Proc. of SPIE Vol. 9512 95121Q-1 


\section{COMB BEAMS AT SPARC-LAB}

The technique used at SPARC to manipulate bunch trains combines the generation of a pulse train at the photocathode with the RF compression ${ }^{3}$ (the so called velocity bunching). The technique used to generate a 2 pulses beam at the cathode relies on a birifrangent crystal, where the input laser pulse is decomposed in two orthogonally polarized pulses with a time separation proportional to the crystal length.

The SPARC velocity bunching technique consists in injecting the beam in an accelerating structure working near the zero crossing of the RF field phase: if the beam is slightly slower than the phase velocity of the RF wave, it slips back to phases where the field is accelerating and at the same time the beam is chirped and compressed up to a point of maximum bunch compression; a further increase of phase delay brings the beam to over-compression and the bunch starts lengthening.

A detailed description of the SPARC layout can be found in Ref. 4, while a description of the machine working point to manipulate bunch trains can be found in Ref.s 5, 6 . It has been shown that to have good separation, one has to operate in over-compression regime, which implies a careful machine optimization to preserve significantly low emittances. On top of that, the experimental reconstruction of the sub-bunches dynamics is not trivial, since the bunch length measurement resolution needed to precisely resolve the subbunches is demanding. Referring to Ref.s5,6), we report here for reader convenience, the major knobs used to achieve set-up the proper liana working points. The results reported later in sec.s ?? have been obtained setting the machine according to the following considerations.

As a general rule of thumb, the bunch separation is roughly twice the total bunch length in overcompression and such separation is completely frozen at the end of the first $3 \mathrm{~m}$ TW section (the SPARC RF compressor). For a given compression phase, the sub-bunches separation increases for increasing the laser pulses distance on the cathode and the whole bunch charge (i.e. the space charge contribution).

The phase distance of the maxima of the sub-bunches compression curves (that is of the sub-bunches minimum length) depends mainly on the pulse distance at the entrance of the RF compressor which, for a given RF gun injection phase, is determined by the space charge: the pulse distance at the RF compressor entrance increases with bunch charge and moving the gun injection phase towards the phase of maximum charge extraction. Simulations show that such a phase separation of the maxima can be inferred from the pulse distance of the on crest beam at the linac end. The sub-bunches minimum length (and thus their maximum compression) is in general different, being identical only in the ideal case of no space charge beams injected with the same energy in the compressor.

The SPARC linac is composed by three $3 \mathrm{~m}$ TW sections after the gun (the first one being the compressor) and the beam injection phase can be varied autonomously for each section; usually the second and the third section are phased to get the maximum energy at the linac exit. By changing the last section phase (i.e. the whole beam energy), only the energy (and not the time) separation is affected; in particular there is no effect on the sub-bunches current distribution.

The difference between the sub-bunches charges at the cathode does not affect the separation at the linac exit (for a given compression phase), while it affects the sub-bunches currents and bunch lengths.

Emittance compensation during the RF compression requires focusing additional to the acceleration one, which in the SPARC case is provided by two $3 \mathrm{~m}$ (independently powered) solenoids embedding the first two TW sections. Typically only the solenoid around the first accelerating section is used at operation values in the range of $270-330 \mathrm{G}$ (for all the data/simulation presented here). Such magnetic field has a negligible effect on the separation, while it affects the emittance (at the linac exit), the sub-bunches bunch length and current (but not the sub-bunches current ratio) and the compression phases corresponding to the single bunch compression curves maxima, which are (more or less) rigidly shifted by varying the magnetic focusing field.

As usual in RF gun based linac, the main knob to control the emittance is the solenoid embedding the gun, affecting the emittance of the whole bunch as well as of the sub-bunches, together with their length and currents. The minimum emittance of the whole bunch, the minimum emittance of each single sub-bunch 
and an equal current in the sub-bunches occur at different values of the gun solenoid field; typically for the SPARC case, these values differ no more than roughly 300G.

As a rule of thumb, the minimum emittance of the whole bunch, the equal current and the minimum spot size at the linac exit occur for close values of the gun solenoid field. On top of that, simulations suggest that the minimum emittance of the whole bunch occurs when the two sub-bunches are radially separated at the linac exit, i.e. one forms a ring around the other in the transverse spot. During machine operation, this condition can be often observed (especially for "well behaved" beams) by letting the beam propagating for roughly 5 meters after the linac without additional (quadrupole) focusing. Therefore such situation was used as starting point for emittance optimization in the experiments discussed below.

A possible procedure, while operating the machine for comb bunches, may be the setting the bunch separation by choosing the compression phase and then the emittance optimization (with the gun solenoid) for a given value of the TW section magnetic focusing field.

All those guidelines were followed during the experiments performed recently with two bunches (200$400 \mathrm{pC}$ ) and four bunches $(200 \mathrm{pC})$ train; the train average energy at the linac exit was varying from 170 to $90 \mathrm{MeV}$, depending on the machine setting, mainly the compression phase (used to tune the sub-bunches distance) and the last section phase (used to tune the sub-bunches energy separation).

The use of the RF compressor in the over-compression regime requires good beam quality (i.e transverse emittance) and overall machine stability. A poor beam emittance would spoil the brilliance and/or would imply a too large beam to be manipulated; the compression phase stability is important to keep the separation in the comb beam constant during operation. To monitor carefully the effect of all the machine parameters, we have also developed a single shot and not-intercepting diagnostics ${ }^{7}$ based on electro-optical sampling.

\section{COMB BEAMS FOR TWO COLORS FEL}

The ability of FELs in the x-rays range to probe matter at the femtosecond time scales is typically exploited in pumpprobe configurations where two pulses with different wavelengths and adjustable time delay are used to investigate the processes occurring during chemical and physical reactions.

Many two color FEL experiments have been recently discussed in literature; the most relevant ones are discussed in Ref. 8. One method, exploited at SPARC-LAB, to generate two-color FEL radiation consists in injecting in the undulator a train of two electron bunches with time and energy separation adjustable by setting the linear accelerator (linac) parameters.

The use of a two energy level electron beam for producing two-color radiation pulses has been studied and tested at SPARC-LAB. ${ }^{2,9}$ Our technique relies on low energy RF compression of a comb-like electron beam, to produce high brightness, sub-picosecond spaced trains of pulses, separated both in energy and time, and capable of producing FEL radiation in the single spike regime. Numerical simulations of the beam dynamics have pointed out the main parameters to achieve the desired beam properties in this two-bunch regime, demonstrating the versatility of the method. ${ }^{5}$ References 2,9 address a specific case which has been investigated in an experiment where the spectral and temporal features of SASE FEL radiation have been fully characterized; the results agreed very well with the simulations of both the electron beam dynamics and the FEL process. Color tunability of the pump and probe pulses that may be obtained changing carefully the working point of the Linac.

This scheme is based on two closely spaced electron bunches generated at the cathode and accelerated off-crest in the following linac; the result is a two beamlets bunch with a small energy difference between the beamlets. Such a bunch train injected in an undulator radiates two distinct SASE (Self Amplified Spontaneous Emission) pulses, whose relative time delay and wavelength difference can be tuned independently by changing the linac working point. A 1-2\% spectral separation can be achieved. Moreover since the whole length of the undulator can be used by both radiation, one can immagine applications requiring high-intensity radiation or exploiting the activation of self-seeding processes.

As in any FEL process, the emission starts from noise, and, whereas the two colors can be single spiked, the radiation is affected by random shot to shot fluctuations both in the time and frequency domains. To 

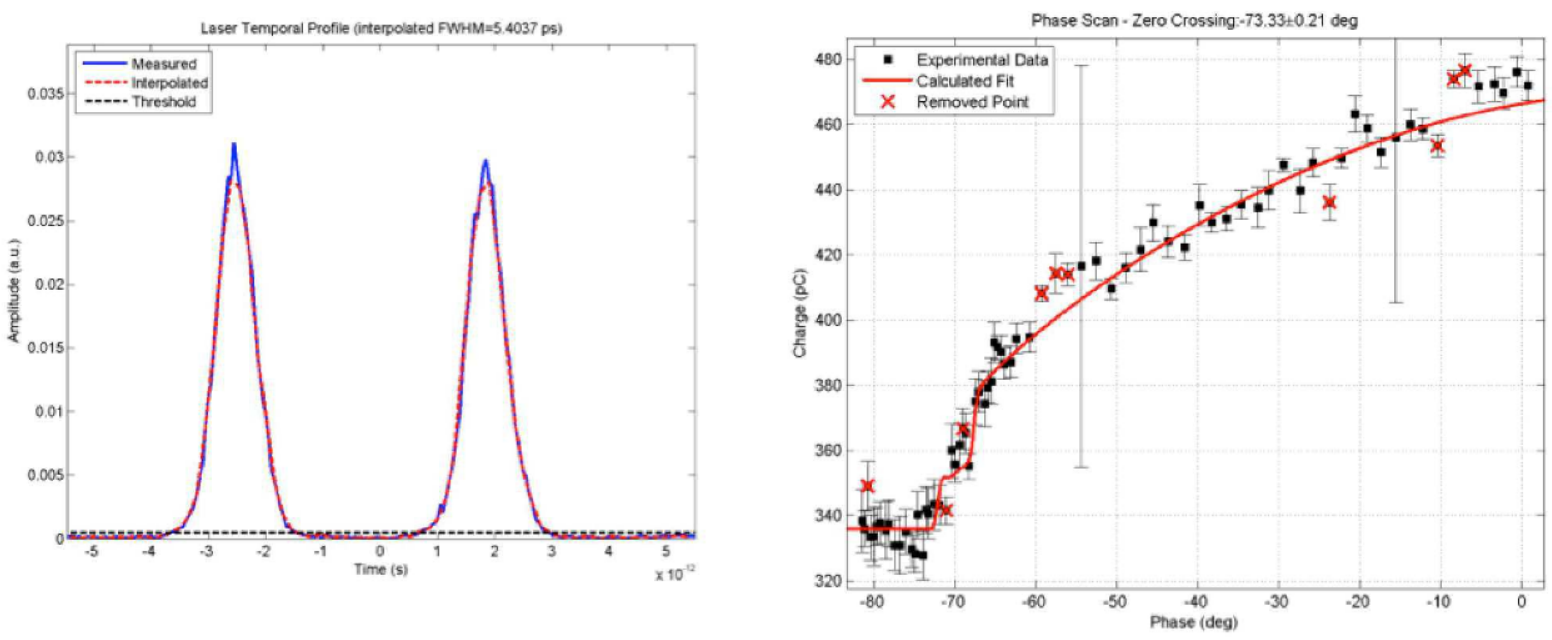

Figure 1. Profile of the laser hitting the photocatode (left picture) and phase scan (right picture).

overcome the intrinsic FEL fluctuations coming from shot-noise, the method has been extended to seeded FEL schemes, where the emission is locked to an external seed laser ${ }^{8}$ and the degree of coherence has been improved.

In this paper we report the operational experience and the main characteristics of the linac working point, in the case of seeded, two color FEL radiation at SPARC-LAB. The results in terms of achieved radiation properties are described elsewhere: full time and spectral informations are there reported. ${ }^{8}$

The two beamlets are obtained by a two-peaked laser pulse impinging the cathode; the time profile of is shown in the left picture of Fig. 1 and the laser FWHM is 5.7ps. The right picture of Fig. 1 shows a plot of extracted charge from the cathode, as a function of the arrival time of the laser with respect to the RF pulse; such arrival time is expressed in degree of the RF and $1 \mathrm{deg}$ is approximately 1 ps since our working frequency is $2.856 \mathrm{GHz}$.

The injection time of the laser with respect to the RF pulse is carefully chosen. The main goal is to exit from the RF gun with the maximum energy, thus reducing the space charge spoling of the beam quality. Such a condition is typically achieved injecting a sigle laser pulse at 30deg after the phase where the emissions starts (i.e. -73deg in Fig. 1). The working point of the linac foresees an injection phase of 33deg; such a value is chosen after a careful numerical optimization with start-to-end beam dynamics simulations and the reason being the two beamlets nature of the beam at the photocathode.

The emission beginning phase is a crucial information to get and it is measured by fitting the chargephase plot, as shown by the red line in the left picture of Fig. 1; for instance in that case the measured phase is -73.33(0.21) deg. The charge emission such a phase is dominated by the laser and the charge profile in proximity of such point resembles the laser profile, having a different derivative and showing a single step since two laser pulses are hitting the cathode. The energy at the gun exit is $5.3 \mathrm{MeV}$ and the total charge is $100 \mathrm{pC}$.

At the end of the linac, after having applied the machine set-up as described in Ref.s 5), we result to have a beam with longitudinal phase space reported in Fig. 2. Two beam are clearly separated in energy $(1 \mathrm{MeV})$ and have charge very close. Due to the different time duration, the current is different and the peak current is above 100A for the lower energy beam and below $80 \mathrm{~A}$ for the higher energy one. The analysis of each beam has been possible due to the energy separation. The energy profile is shown in the left picture of Fig. 2.

In order to achieve optimal beam transport in the undulators, the matching conditions are imposed from the Twiss properties of the whole beam (and not on the transverse dynamics of the single beamlets). 

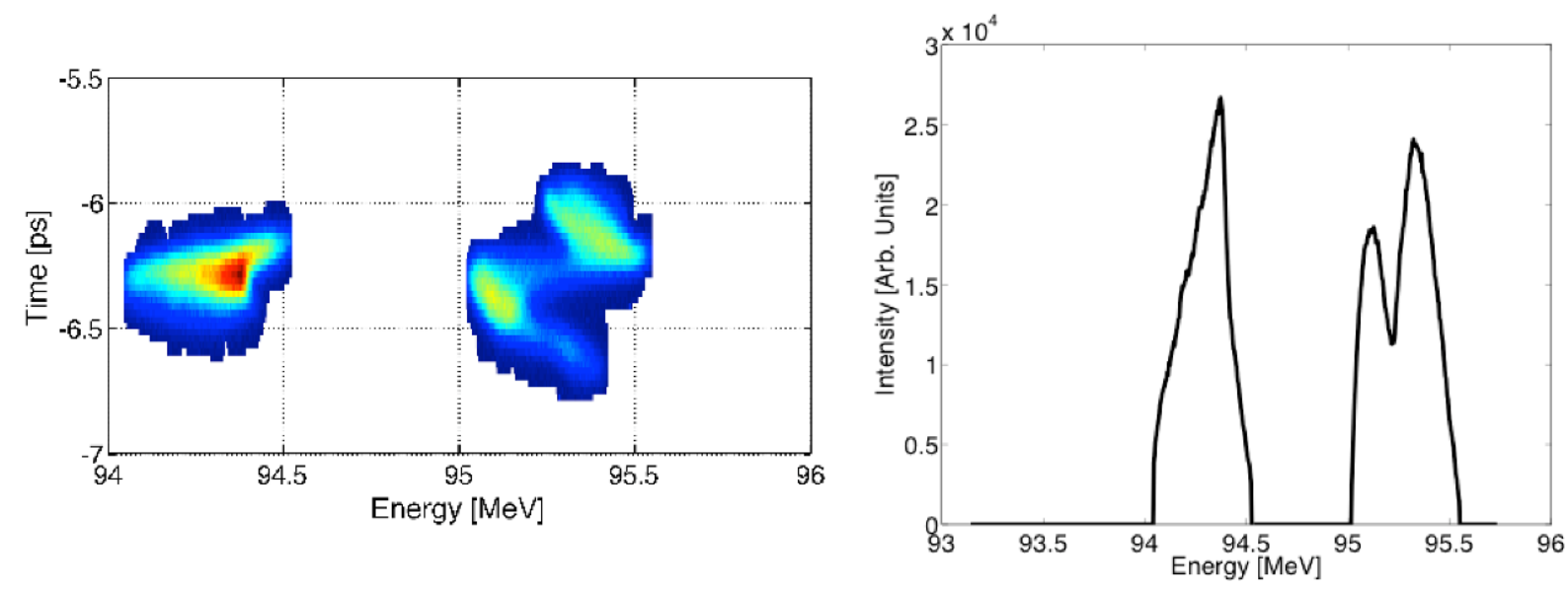

Figure 2. Longitudinal phase space for the compressed bunch train (left picture) and energy profile (right picture). The total charge is $100 \mathrm{pC}$.
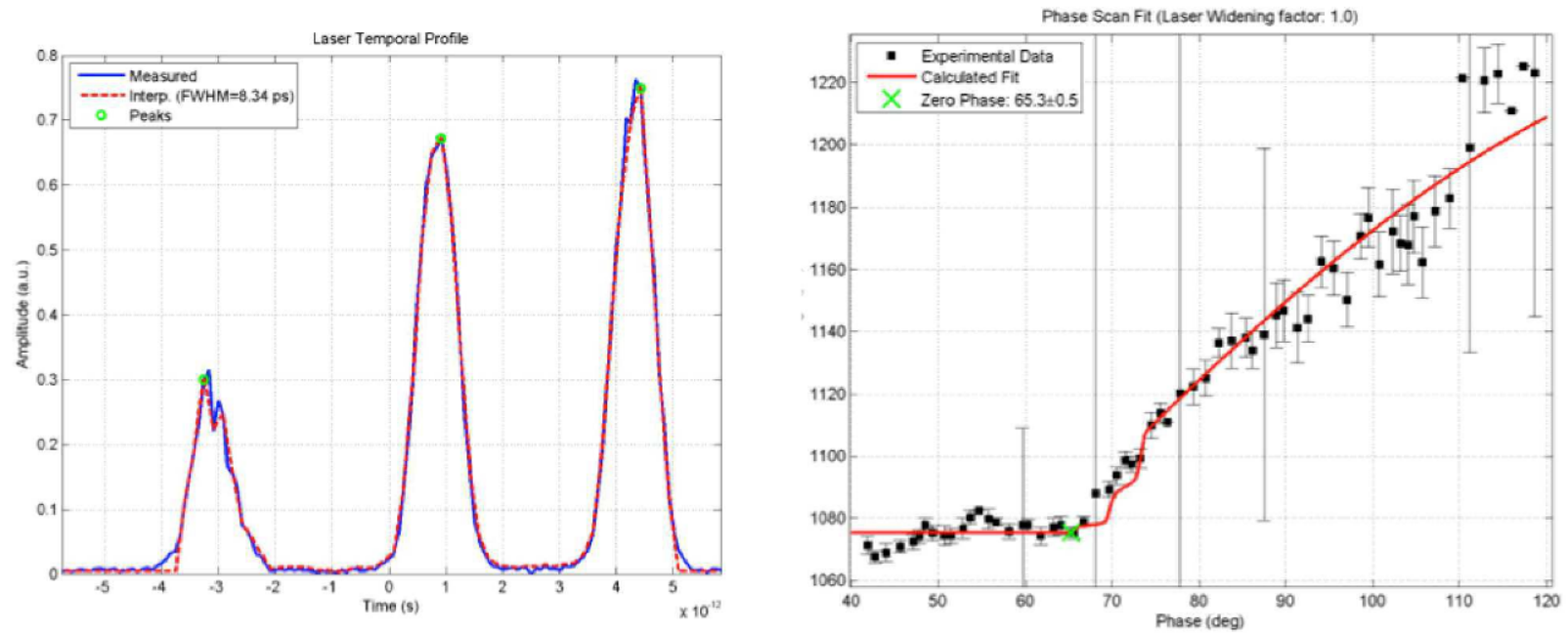

Figure 3. Profile of the laser hitting the photocatode (left picture) and phase scan (right picture).

By theoretical considerations and according to our operation experience, we have found that setting the undulator channel to match the whole beam, assure lasing of the two beamlets. The total emittance is around $2 \mu \mathrm{m}$ and it has been achieved after a careful optimization of the solenoid around the RF gun and one around the first traveling wave section of the SPARC linac.

\section{COMB BEAMS FOR PLASMA ACCELERATION}

In this section, we report on the generation of three beamlets bunch train. The three beamlets are again obtained by a three-peaked laser pulse impinging the cathode; the time profile of is shown in the left picture of Fig. 3 and the laser FWHM is 8.34 ps. The right picture of Fig. 3 shows a plot of extracted charge from the cathode, as a function of the arrival time of the laser with respect to the RF pulse.

The injection phase in this case is strictly 30deg after the phase where the emissions starts (i.e. 65.5deg in Fig. 3); such a value has been assessed with dedicated start-to-end beam dynamics simulations. The charge 

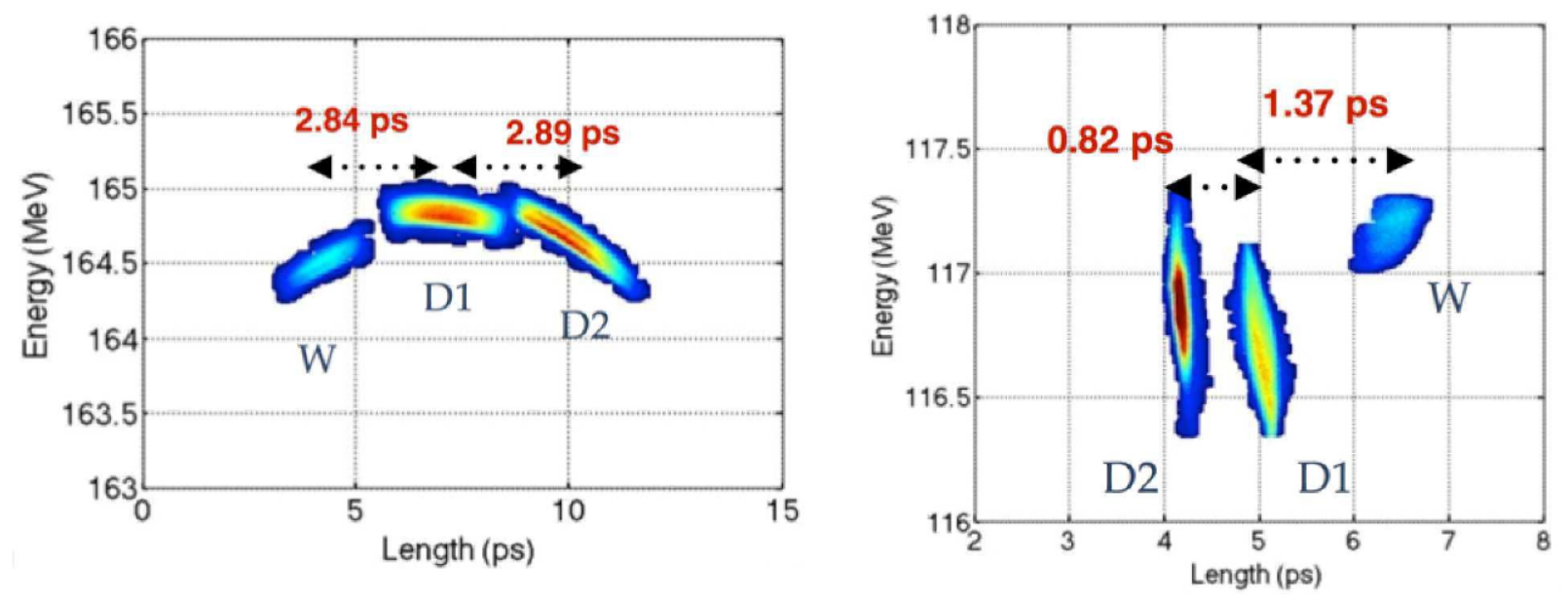

Figure 4. Longitudinal phase space for the uncompressed bunch train (left picture) and the over compresses one (right picture) at the linac exit. The two driving bunches are labeled "D1" and "D2", while "W" stands for witness bunch.

emission profile in the vicinity of the beginning of the charge emission, resembles again the laser profile showing a two-steps shape (corresponding to three laser pulses). The energy at the gun exit is $5.34 \mathrm{MeV}$ and the total charge is $97 \mathrm{pC}$.

Figure 4 shows the longitudinal phase space at the maximum energy (on-crest, left picture) and at the working point (over-compression, right picture). The configuration of two drivers (namely D1 and D2) and a witness (W) is clear. In PWFA, the driver will resonantly excite the plasma, allowing the witness to be accelerated. The distance between the drivers must be one plasma wavelength (about 0.8ps in our case) while the witness must be distant 1.5 plasma wavelength (i.e about $1.2 \mathrm{ps}$ in our case).

The achieved emittance in the on-crest case is about $1 \mu \mathrm{m}$ in both planes at $164 \mathrm{MeV}$; the two drivers have about the same charge (around $40 \mathrm{pC}$ ) and the witness has a charge of $20 \mathrm{pC}$. Concerning the bunch lengths, the drivers are around $700 \mathrm{fs}$, while the witness is around $500 \mathrm{fs}$.

The same charge are conserved also in the over-compression working point at $117 \mathrm{MeV}$. The beamlets are shorter, being the drivers in the range of $100 \mathrm{fs}$ and the witness about $200 \mathrm{fs}$. The transverse emittance shows an asymmetry between the two planes, probably due to the the some misalignment of the beamlets. Anyhow the square root of the product of the emittances in the two planes is around $4 \mu \mathrm{m}$. Another interesting feature shown experimentally is the capability of continuously moving the witness bunch by delaying the third optical pulse hitting in the cathode.

\section{PERSPECTIVES ON THE NEXT SPARC OPERATIONS AND CONCLUSIONS}

The goal of the ongoing and next beam studies at SPARC is the demonstration of plasma acceleration of high brightness beam. The SPARC FEL undulators will be used as "diagnostics" elements to prove the brightness of the beam. The SPARC-LAB linac will undergo a maximum energy upgrade with the installation of a C-band accelerating section ${ }^{10}$ which have an higher accelerating gradient and, being are more compact, will free the space in the beam line to be used for PWFA experimental chamber.

In this paper we have shown some relevant beam configuration achieved at SPARC-LAB manipulating the bunch trains. The manipulations uses peculiarity of the SPARC linac, such as velocity bunching with focusing solenoids. The main experiments addressed multicolor FEL and plasma acceleration experiment preparation. 


\section{ACKNOWLEDGMENTS}

This work has been partially funded by the Italian Minister of Research in the framework of FIRB - Fondo per gli Investimenti della Ricerca di Base, Project no. RBFR12NK5K and EU Commission in the Seventh Framework Program, Grant Agreement 312453 - EuCARD-2.

\section{REFERENCES}

[1] Chiadroni, E., "The sparc-lab thz source driven by high-brightness electron beams," Nuovo Cimento della Societa Italiana di Fisica C 37(4), 349-354 (2014).

[2] Petrillo, V., Anania, M. P., Artioli, M., Bacci, A., Bellaveglia, M., Chiadroni, E., Cianchi, A., Ciocci, F., Dattoli, G., Di Giovenale, D., Di Pirro, G., Ferrario, M., Gatti, G., Giannessi, L., Mostacci, A., Musumeci, P., Petralia, A., Pompili, R., Quattromini, M., Rau, J. V., Ronsivalle, C., Rossi, A. R., Sabia, E., Vaccarezza, C., and Villa, F., "Observation of time-domain modulation of free-electron-laser pulses by multipeaked electron-energy spectrum," Phys. Rev. Lett. 111, 114802 (Sep 2013).

[3] Ferrario, M., Alesini, D., Bacci, A., Bellaveglia, M., Boni, R., Boscolo, M., Castellano, M., Chiadroni, E., Cianchi, A., Cultrera, L., Di Pirro, G., Ficcadenti, L., Filippetto, D., Fusco, V., Gallo, A., Gatti, G., Giannessi, L., Labat, M., Marchetti, B., Marrelli, C., Migliorati, M., Mostacci, A., Pace, E., Palumbo, L., Quattromini, M., Ronsivalle, C., Rossi, A., Rosenzweig, J., Serafini, L., Serluca, M., Spataro, B., Vaccarezza, C., and Vicario, C., "Experimental demonstration of emittance compensation with velocity bunching," Physical Review Letters 104(5) (2010).

[4] Ferrario, M., Alesini, D., Anania, M., Bacci, A., Bellaveglia, M., Bogdanov, O., Boni, R., Castellano, M., Chiadroni, E., Cianchi, A., Dabagov, S., Martinis, C., Giovenale, D., Pirro, G., Dosselli, U., Drago, A., Esposito, A., Faccini, R., Gallo, A., Gambaccini, M., Gatti, C., Gatti, G., Ghigo, A., Giulietti, D., Ligidov, A., Londrillo, P., Lupi, S., Mostacci, A., Pace, E., Palumbo, L., Petrillo, V., Pompili, R., Rossi, A., Serafini, L., Spataro, B., Tomassini, P., Turchetti, G., Vaccarezza, C., Villa, F., Dattoli, G., Palma, E., Giannessi, L., Petralia, A., Ronsivalle, C., Spassovsky, I., Surrenti, V., Gizzi, L., Labate, L., Levato, T., and Rau, J., "Sparc-lab present and future," Nuclear Instruments and Methods in Physics Research, Section B: Beam Interactions with Materials and Atoms 309, 183-188 (2013).

[5] Mostacci, A., Alesini, D., Antici, P., Bacci, A., Bellaveglia, M., Boni, R., Castellano, M., Chiadroni, E., Cianchi, A., Di Pirro, G., Drago, A., Ferrario, M., Gallo, A., Gatti, G., Ghigo, A., Marchetti, B., Migliorati, M., Pace, E., Palumbo, L., Petrillo, V., Ronsivalle, C., Rossi, A. R., Serafini, L., Spataro, B., and Vaccarezza, C., "Advanced beam manipulation techniques at sparc," in [IPAC 2011 - 2nd International Particle Accelerator Conference], 2877-2881 (2011).

[6] Mostacci, A., "Comb beam for particle-driven plasma-based accelerators," Nuovo Cimento della Societa Italiana di Fisica C 37(4), 355-364 (2014).

[7] Pompili, R., Cianchi, A., Alesini, D., Anania, M., Bacci, A., Bellaveglia, M., Castellano, M., Chiadroni, E., Giovenale, D. D., Pirro, G. D., Gatti, G., Giorgianni, F., Ferrario, M., Lupi, S., Massimo, F., Mostacci, A., Rossi, A., Vaccarezza, C., and Villa, F., "First single-shot and non-intercepting longitudinal bunch diagnostics for comb-like beam by means of electro-optic sampling," Nuclear Instruments and Methods in Physics Research Section A: Accelerators, Spectrometers, Detectors and Associated Equipment 740(0), 216 - 221 (2014). Proceedings of the first European Advanced Accelerator Concepts Workshop 2013.

[8] Petralia, A., Anania, M. P., Artioli, M., Bacci, A., Bellaveglia, M., Carpanese, M., Chiadroni, E., Cianchi, A., Ciocci, F., Dattoli, G., Di Giovenale, D., Di Palma, E., Di Pirro, G.and Ferrario, M., Giannessi, L., Innocenti, L., Mostacci, A., Petrillo, V., Pompili, R., Rau, J. V., Ronsivalle, C., Rossi, A. R., Sabia, E., Shpakov, V., Vaccarezza, C., and Villa, F., "Two-color radiation generated in a seeded free-electron laser with two electron beams," submitted to Phys. Rev. Lett. (Mar 2014).

[9] Ronsivalle, C., Anania, M. P., Bacci, A., Bellaveglia, M., Chiadroni, E., Cianchi, A., Ciocci, F., Dattoli, G., Giovenale, D. D., Pirro, G. D., Ferrario, M., Gatti, G., Giannessi, L., Mostacci, A., Musumeci, P., Palumbo, L., Petralia, A., Petrillo, V., Pompili, R., Rau, J. V., Rossi, A. R., Vaccarezza, C., and Villa, F., "Large-bandwidth two-color free-electron laser driven by a comb-like electron beam," New Journal of Physics 16(3), 033018 (2014). 
[10] Alesini, D., Boni, R., Di Pirro, G., Di Raddo, R., Ferrario, M., Gallo, A., Lollo, V., Marcellini, F., Palumbo, L., Spizzo, V., Mostacci, A., Campogiani, G., Persichelli, S., Enomoto, A., Higo, T., Kakihara, K., Kamitani, T., Matsumoto, S., Sugimura, T., Yokoyama, K., and Verd-Andrs, S., "The c-band accelerating structures for sparc photoinjector energy upgrade," Journal of Instrumentation 8(5) (2013). 